\title{
Energy decay rate of multidimensional inhomogeneous Landau-Lifshitz-Gilbert equation and Schrödinger map equation on the sphere
}

\author{
Penghong Zhong ${ }^{1 *} \mathbb{D}$, Chao Zhang ${ }^{1}$ and Fengong $\mathrm{Wu}^{1}$
}

*Correspondence:

penghongzhong@yahoo.com 1 Department of Mathematics, Guangdong University of Education, Guangzhou, China

\section{Springer}

\begin{abstract}
We consider the multidimensional dimensional inhomogeneous Landau-Lifshitz-Gilbert (ILLG) equation and its degenerate case, the Schrödinger map equation. We investigate the special solutions (under large initial values) and their energy property of the ILLG and Schrödinger map equations. Until now, we had not seen a paper presenting an explicit dynamic solution of the multidimensional ILLG. Using the stereographic method, an equivalent equation of ILLG is obtained. Based on this equivalent system, we obtain some exact solutions of the ILLG equation and present some implicit solutions of the Schrödinger map equation. Based on these solutions, by a careful estimation we give the decay rate of energy density.
\end{abstract}

Keywords: Landau-Lifshitz-Gilbert equation; Schrödinger map equation; Decay rate

\section{Introduction}

Long-wavelength spin motions in diverse ferromagnetic structures are commonly described by the Landau-Lifshitz-Gilbert equation (or the LLG equation), which was first derived by Landau and Lifshitz [14]. The LLG equation is a very fundamental equation in describing the evolution for the magnetization in ferromagnetic materials such as NavierStokes equations in fluid dynamics. Without the effects of anisotropy and external magnetic field, the LLG equation can be written as

$$
\frac{\partial}{\partial t} S=\alpha S \times \Delta S-\beta S \times(S \times \Delta S),
$$

where $S=\left(S_{1}, S_{2}, S_{3}\right), S \in S^{2} \hookrightarrow R^{3}, \alpha^{2}+\beta^{2}=1(\alpha \geq 0$ and $\beta \geq 0)$, and $\times$ denotes the cross product.

The LLG equation is a mixture of two famous equations, the Schrödinger map equation $(\alpha=1)$ and the harmonic map heat flow $(\beta=1)$. The term multiplied with $\alpha$ represents the exchange interaction. From a physical point of view, the exchange interaction constitutes an indispensable part of the LLG system. However, the $\beta$-term denotes to the Gilbert damping considered as a dissipation factor in the LLG equation.

(c) The Author(s) 2018. This article is distributed under the terms of the Creative Commons Attribution 4.0 International License (http://creativecommons.org/licenses/by/4.0/), which permits unrestricted use, distribution, and reproduction in any medium, provided you give appropriate credit to the original author(s) and the source, provide a link to the Creative Commons license, and indicate if changes were made. 
In the setting of the LLG equation, of particular importance is to consider the effect of inhomogeneity on the spin system. This extension is somewhat like the the inhomogeneous Schrödinger map equation proposed by Balakrishnan [1]. If the dissipation and inhomogeneity are considered in LLG, (1) can be generalized into the ILLG equation. Motivated by these considerations, in this work, we consider the $n$-dimensional ILLG equations

$$
\begin{aligned}
\frac{\partial}{\partial t} S= & \alpha[q(t, \vec{x}) S \times \Delta S+S \times(\nabla q(t, \vec{x}) \nabla S)] \\
& -\beta S \times[q(t, \vec{x}) S \times \Delta S+S \times(\nabla q(t, \vec{x}) \nabla S)],
\end{aligned}
$$

where the scalar function $q(t, \vec{x})$ is the inhomogeneous term.

In the setting of the LLG equation (1), we list several known results. Liu [13] proved that the concentration set for fixed $t$ is an $H^{m-2}$-rectifiable set for almost all $t$ for LLG. For a smallness initial condition on the gradient, the global well-posedness results for (1) have been established in $n \geq 2$ by Melcher [17]. Some further works about the smallness condition for well-posedness of (1) were done by Lin, Lai, and Wang [15] in the Morrey space. The smallness condition does not always mean that the global solution exists. Ding and Wang [7] proved that the solution of (1) develops a finite time singularity in dimensions 3 or 4 under small special initial data. In the critical dimension $n=2$, (1) contains a blowup solution, and the exact blowup rate is predicted by formal analysis [26] for some special large data. If $\alpha=0$, then LLG degenerates into a harmonic map heat flow. Similarly, comparing a harmonic map heat flow with some general harmonic system [22, 32-34], it is clear that the mapping one is more difficult to deal with than a nonmapping system. The most difficulty of a mapping system is caused by the curvature flow of the Riemannian manifolds.

The results on the Schrödinger map equation are much more fruitful than those on the LLG equation. There are a lot fruitful results on the existence, uniqueness, and the blowup property and soliton solution of the nonlinear Schrödinger equation [11, 19, 24, 31]. Even for the fractional Schrödinger equations, there are some recent fruitful results $[2,8,18$, 20]. However, very few results can be seen for the Schrödinger map equation, and some further work still needs to be done. We refer the reader to [25] for some results about the local existence theorem. Some progress of small initial data existence results can be found in [4] and [3] for $n \geq 2$. Especially, the classical solution with small energy is global in time for the radial case [4]. For some special large initial data, the possibility of a finite time blowup and the blowup rate has been proved [21, 23]. In 2008, Huh [10] constructed infinite energy explicit blowup solutions for the modified Schrödinger map equation. Ding [5] constructed an infinite energy blowup solution for the Schrödinger map equation on a hyperbolic target.

In spite of these developments on the LLG equation and Schrödinger map equation, little progress has been made in the case of ILLG equation. Comparing to many existence results of the homogeneous case, in which $q(t, \vec{x})$ (shortly, $q$ ) is a constant, the inhomogeneous case is not so clear (even the local solution). However, these existence (or blowup) results of the LLG equation or Schrödinger map equation are expected to be extended to the inhomogeneous case. 
For $\beta=0$, Daniel et al. [6] analyzed the singularity structure of solutions of (2). More exactly, (2) identified the integrable model under the inhomogeneity as follows:

$$
q=\frac{\varepsilon_{1}}{r^{2(n-1)}}+\frac{\varepsilon_{2}}{r^{n-2}}
$$

where $r$ is the radial spatial variable.

The Lax pair of this integrable case was found, and some soliton like solutions are also presented in [6]. However, Li and Wang [16] proved that blowup occurs in some specific format of the inhomogeneous term. In fact, their did not provide an exact form of the solution of the inhomogeneous Schrödinger map equation. If $q$ takes some decay forms, then this equation contains some finite-time blowup solutions such as the solutions first presented in $[28,29]$. Similarly, this equation ill also develops some finite-time blowup solution on the hyperbolic target [30]. More clearly, in [28, 29], the authors provided a solution of the form

$$
S(t, \vec{x})=\left(\begin{array}{c}
\sin \left(F_{1}(\vec{x})\right) \cos \left(\frac{F_{2}(\vec{x})}{G_{1}(t)}\right) \\
\sin \left(F_{1}(\vec{x})\right) \sin \left(\frac{F_{2}(\vec{x})}{G_{1}(t)}\right) \\
\cos \left(F_{1}(\vec{x})\right)
\end{array}\right)
$$

where $F_{1}(\vec{x})$ and $F_{2}(\vec{x})$ are functions of $\vec{x}$, and $G_{1}(t)$ is a function of $t$ such that $G_{1}(t) \rightarrow$ $+\infty$ as $t \rightarrow T$ ( $T$ is a positive constant).

These finite-time blowup solutions indicate that some special solutions of the equation can develop a finite-time singularity from smooth initial data with finite energy in finite (or infinite) spatial domain. It is a natural question to ask whether the ILLG equation contains some blowup or nonblowup solution. However, we cannot obtain any explicit or implicit solution under the constraint of (3). Therefore, in this paper, we are devoted to solve the ILLG equation under another type of solution,

$$
S(t, \vec{x})=\left(\begin{array}{c}
\left.\sin \left(F_{1}(\vec{x})\right) \cos \left(F_{2}(\vec{x})+G_{1}(t)\right)\right) \\
\left.\sin \left(F_{1}(\vec{x})\right) \sin \left(F_{2}(\vec{x})+G_{1}(t)\right)\right) \\
\cos \left(F_{1}(\vec{x})\right)
\end{array}\right),
$$

where $F_{1}(\vec{x})$ and $F_{2}(\vec{x})$ are functions of $\vec{x}$, and $G_{1}(t)$ is a function of $t$.

We use the variable separation ansatz (4) to construct the solution of ILLG equation in Sect. 3. At the same time, we employ this form to construct an implicit solution of the Schrödinger map equation in Sect. 4.

Up to now, we have never seen any paper discussing how to exactly solve the ILLG equation. As far as we know, even for the LLG equation and Schrödinger map equation, the exact treatment is very scarce. The ILLG and Schrödinger map equations are nonintegrable systems in most cases. Based on the property of the Hamiltonian systems [39-41], the bifurcation structure of the general integrable systems and autonomous differential systems [35-38] are clear. However, the property of the nonintegrability of a system needs to be further explored. In this paper, we present some special solutions of the ILLG equation and Schrödinger map equation and discuss their properties to enrich the solutions of these equations. It is well known that the main barrier to the ILLG equation and Schrödinger 
map equation is that there is no energy monotonicity inequality for them. Hence, it is difficult to determine how the solution will evolve after a long time or in the remote area. We present some results, which may be expected to result in a better understanding of these two systems. More exactly, we study the special solutions in the form of (4) and present some explicit or implicit structures of it, which will be useful in studying their properties.

Based on the smallness initial condition, we can prove the global existence of weak (or even smooth) solutions of the Schrödinger map equation (or even ILLG). However, the question of regularity and uniqueness of weak solutions is a delicate question depending on the initial data. In this paper, we study some special large initial data solutions. For the ILLG, some properties of the global type solution are clearer because of their straightforward and exact form. We also present some implicit solution for the Schrödinger map equation. At the same time, we analyze a type of special large initial data solution, which indicates a global solution.

The plan of the paper is as follows. In Sect. 2, we construct the equation of motion to represent the ILLG equation and then obtain an equivalent generalized nonlinear modification equation by the stereographic projection method. In Sect. 3, we construct the solution of this equivalent system and deduce the solution of the ILLG equation and analyze the energy density of these two equivalent equations. We study the variable separation solutions under the cylindrical coordinates and the normal coordinates in Sect. 4. In this section, we also carry out the decay analysis to identify the asymptotic behavior of the energy density.

\section{Equivalent equation of ILLG equation}

In this section, we deduce an equivalent system of (2), which is helpful in constructing its solution. As we know, the Hasimoto transformation is usually employed to convert some geometrical partial differential equation to another complex equation. However, it is always difficult to avoid the emergence of some integral term in the deduced new system. Always, it is difficult to solve this new deduced system due to the new integral term difficult to deal with. The stereographic projection method avoids some defects such as the appearing of integral section when we do the equivalent transformation between the two systems. Furthermore, if we obtain a solution of the new deriving equation, then we can usually obtain the solution of the original equation by the opposite transformation of the stereographic projection. However, it is difficult to do this opposite process for the equivalent deriving system under the Hasimoto transformation. Considering this situation, we use the stereographic projection to deduce the equivalent system that does not contain a nonlocal term. First, we calculate the expanded form of (2):

$$
\begin{aligned}
& \alpha \Psi_{1}-\beta\left(S_{2} \Psi_{3}-S_{3} \Psi_{2}\right)-S_{1 t}=0, \\
& \alpha \Psi_{2}-\beta\left(-S_{1} \Psi_{3}+S_{3} \Psi_{1}\right)-S_{2 t}=0, \\
& \alpha \Psi_{3}-\beta\left(S_{1} \Psi_{2}-S_{2} \Psi_{1}\right)-S_{3 t}=0,
\end{aligned}
$$

where

$$
\begin{aligned}
& \Psi_{1}=q\left(S_{2} \Delta S_{3}-S_{3} \Delta S_{2}\right)+\nabla q\left(S_{2} \nabla S_{3}-S_{3} \nabla S_{2}\right), \\
& \Psi_{2}=q\left(-S_{1} \Delta S_{3}+S_{3} \Delta S_{1}\right)+\nabla q\left(-S_{1} \nabla S_{3}+S_{3} \nabla S_{1}\right),
\end{aligned}
$$




$$
\Psi_{3}=q\left(S_{1} \Delta S_{2}-S_{2} \Delta S_{1}\right)+\nabla q\left(S_{1} \nabla S_{2}-S_{2} \nabla S_{1}\right)
$$

The couple system (5)-(10) is complicated. If we could simplify it, the resolving progress would become much simpler. As we will see, (5)-(10) will adopt another single complex form. We definite

$$
W=\frac{S_{1}+i S_{2}}{1+S_{3}}
$$

Furthermore, $\bar{W}$ denotes the conjugate complex numbers of $W$; the real and imaginary parts of the complex number $W$ are $\Re(W)$ and $\Im(W)$, respectively. The spin vector $S$ of LLG equation (2) is laid on $\mathbb{S}^{2}$. Hence, we can use the conversion

$$
\left(S_{1}, S_{2}, S_{3}\right)=\left(\frac{2 \Re(W)}{1+W \bar{W}}, \frac{2 \Im(W)}{1+W \bar{W}}, \frac{1-W \bar{W}}{1+W \bar{W}}\right)
$$

The derivative of each component of $S_{1}$ is

$$
\begin{aligned}
& S_{1 t}=\Phi\left[-W^{2} \overline{W_{t}}-(\bar{W})^{2} W_{t}+W_{t}+\overline{W_{t}}\right], \\
& \nabla S_{1}=\Phi\left[-W^{2} \overline{\nabla W}-(\bar{W})^{2} \nabla W+\nabla W+\overline{\nabla W}\right],
\end{aligned}
$$

where $\Phi=(1+W \bar{W})^{-2}$.

Similarly, the first-order derivative of $S_{2}$ is

$$
\begin{aligned}
& S_{2 t}=i \Phi\left[W^{2} \overline{W_{t}}-(\bar{W})^{2} W_{t}-W_{t}+\overline{W_{t}}\right] \\
& \nabla S_{2}=i \Phi\left[W^{2} \overline{\nabla W}-(\bar{W})^{2} \nabla W-\nabla W+\overline{\nabla W}\right]
\end{aligned}
$$

and the derivative of $S_{3}$ is

$$
\begin{aligned}
& S_{3 t}=-2 \Phi\left(W_{t} \bar{W}+W \overline{W_{t}}\right), \\
& \nabla S_{3}=-2 \Phi(\nabla W \bar{W}+W \overline{\nabla W}) .
\end{aligned}
$$

Setting $\langle A, B\rangle=A \cdot B$ and $\langle A\rangle=A \cdot A$, the derivatives of (13), (15), and (17) are

$$
\begin{aligned}
\Delta S_{1}= & \Phi\left[-W^{2} \overline{\Delta W}-(\bar{W})^{2} \Delta W+\Delta W+\overline{\Delta W}\right] \\
& +2 \Phi^{3 / 2}\left[\langle\nabla W\rangle(\bar{W})^{3}+\langle\overline{\nabla W}\rangle W^{3}-\langle\nabla W\rangle \bar{W}\right. \\
& -2\langle\nabla W, \overline{\nabla W}\rangle W-2\langle\nabla W, \overline{\nabla W}\rangle \bar{W}-\langle\overline{\nabla W}\rangle W], \\
\Delta S_{2}= & i \Phi\left[\overline{\Delta W} W^{2}-(\bar{W})^{2} \Delta W+\overline{\Delta W}-\Delta W\right] \\
& +2 i \Phi^{3 / 2}\left[-\langle\overline{\nabla W}\rangle W^{3}+(\bar{W})^{3}\langle\nabla W\rangle-\langle\overline{\nabla W}\rangle W\right. \\
& -2\langle\overline{\nabla W}, \nabla W\rangle \bar{W}+2\langle\overline{\nabla W}, \nabla W\rangle W+\bar{W}\langle\nabla W\rangle],
\end{aligned}
$$

and

$$
\Delta S_{3}=-2 \Phi[W \overline{\Delta W}+\Delta W \bar{W}]+4 \Phi^{3 / 2}\left[W^{2}\langle\overline{\nabla W}\rangle+W \bar{W}\langle\nabla W, \overline{\nabla W}\rangle\right.
$$




$$
\left.+(\bar{W})^{2}\langle\nabla W\rangle-\langle\nabla W, \overline{\nabla W}\rangle\right]
$$

respectively.

Substituting (11)-(20) into (5), the complex equation of $W$ is

$$
\begin{aligned}
W_{t} \bar{W} & +W \bar{W}_{t} \\
= & -(\alpha-\beta i) i q\left[W \overline{\Delta W}-\Delta W \bar{W}+2 \frac{\langle\nabla W\rangle(\bar{W})^{2}}{W \bar{W}+1}-2 \frac{\langle\overline{\nabla W}\rangle W^{2}}{W \bar{W}+1}\right] \\
& -(\alpha-\beta i) i \nabla q \cdot(W \overline{\nabla W}-\nabla W \bar{W}),
\end{aligned}
$$

which can be simplified as

$$
\begin{aligned}
\Re\left(W_{t} \bar{W}\right)= & -(\alpha-\beta i) i\left[-i q \Im(\Delta W \bar{W})+i q \Im\left(\frac{2\langle\nabla W\rangle(\bar{W})^{2}}{W \bar{W}+1}\right)\right. \\
& -i \nabla q \cdot \Im(\nabla W \bar{W})]
\end{aligned}
$$

or

$$
\begin{aligned}
\Re\left(\bar{W}_{t} W\right)= & -(\alpha-\beta i) i\left[i q \Im(\Delta \bar{W} W)-i q \Im\left(\frac{2\langle\nabla \bar{W}\rangle(W)^{2}}{W \bar{W}+1}\right)\right. \\
& +i \nabla q \cdot \Im(\nabla \bar{W} W)] .
\end{aligned}
$$

Similarly to (21) or (22), (6) transforms into

$$
\begin{aligned}
\overline{W_{t}}\left(W^{2}-1\right)+W_{t}\left(\bar{W}^{2}-1\right) \\
=-(\alpha-\beta i) i q\left[\overline{\Delta W}\left(W^{2}-1\right)-\Delta W\left(\bar{W}^{2}-1\right)-2 \frac{\langle\overline{\nabla W}\rangle W\left(W^{2}-1\right)}{1+W \bar{W}}\right. \\
\left.\quad+2 \frac{\langle\nabla W\rangle \bar{W}\left(\bar{W}^{2}-1\right)}{1+W \bar{W}}\right]-(\alpha-\beta i) i \nabla q \cdot\left[\overline{\nabla W}\left(W^{2}-1\right)-\nabla W\left(\bar{W}^{2}-1\right)\right]
\end{aligned}
$$

which can be rearranged as

$$
\begin{aligned}
\Re\left(W_{t}\left(\bar{W}^{2}-1\right)\right) \\
=-(\alpha-\beta i) i\left[-i q \Im\left(\Delta W\left(\bar{W}^{2}-1\right)\right)+q i \Im\left(\frac{2\langle\nabla W\rangle \bar{W}\left(\bar{W}^{2}-1\right)}{W \bar{W}+1}\right)\right. \\
\left.\quad-i \nabla q \cdot \Im\left(\nabla W\left(\bar{W}^{2}-1\right)\right)\right]
\end{aligned}
$$

or

$$
\begin{aligned}
& \Re\left(\bar{W}_{t}\left(W^{2}-1\right)\right) \\
& \quad=-(\alpha-\beta i) i\left[i q \Im\left(\Delta \bar{W}\left(W^{2}-1\right)\right)-i q \Im\left(\frac{2\langle\nabla \bar{W}\rangle W\left(W^{2}-1\right)}{W \bar{W}+1}\right)\right.
\end{aligned}
$$




$$
\left.+i \nabla q \cdot \Im\left(\nabla \bar{W}\left(W^{2}-1\right)\right)\right]
$$

Similarly, we obtain the equivalent equation of (7)

$$
\begin{aligned}
\overline{W_{t}}\left(W^{2}+1\right)-W_{t}\left(\bar{W}^{2}+1\right) \\
=-(\alpha-\beta i) i q\left[\overline{\Delta W}\left(W^{2}+1\right)+\Delta W\left(\bar{W}^{2}+1\right)-2 \frac{\langle\overline{\nabla W}\rangle W\left(W^{2}+1\right)}{1+W \bar{W}}\right. \\
\left.\quad-2 \frac{\langle\nabla W\rangle \bar{W}\left(\bar{W}^{2}+1\right)}{1+W \bar{W}}\right]-(\alpha-\beta i) i \nabla q \cdot\left[\overline{\nabla W}\left(W^{2}+1\right)+\nabla W\left(\bar{W}^{2}+1\right)\right],
\end{aligned}
$$

which can be rewritten as

$$
\begin{aligned}
\Im & \left(W_{t}\left(\bar{W}^{2}+1\right)\right) \\
= & -(\alpha-\beta i) i\left[-i q \Re\left(\Delta W\left(\bar{W}^{2}+1\right)\right)+i q \Re\left(\frac{2\langle\nabla W\rangle \bar{W}\left(\bar{W}^{2}+1\right)}{W \bar{W}+1}\right)\right. \\
& \left.\quad-i \nabla q \cdot \Re\left(\nabla W\left(\bar{W}^{2}+1\right)\right)\right]
\end{aligned}
$$

or

$$
\begin{aligned}
-\Im & \left(\bar{W}_{t}\left(W^{2}+1\right)\right) \\
= & -(\alpha-\beta i) i\left[-i q \Re\left(\Delta \bar{W}\left(W^{2}+1\right)\right)+i q \Re\left(\frac{2\langle\nabla \bar{W}\rangle W\left(W^{2}+1\right)}{W \bar{W}+1}\right)\right. \\
& \left.-i \nabla q \cdot \Re\left(\nabla \bar{W}\left(W^{2}+1\right)\right)\right] .
\end{aligned}
$$

According to (21), (23), and (25) (or (22), (24), and (26)), we obtain the complex equation of $W$ :

$$
-(\alpha+\beta i) i W_{t}=q \Delta W+\nabla q \cdot \nabla W-\frac{2 q \bar{W}}{1+|W|^{2}}\langle\nabla W\rangle
$$

Under the cylindrical coordinates, it is not difficult to verify that (27) satisfies

$$
-(\alpha+\beta i) i W_{t}=q\left(W_{r r}+\frac{n-1}{r} W_{r}\right)-\frac{2 q \bar{W}\left\langle W_{r}\right\rangle}{1+|W|^{2}}+q_{r} W_{r}
$$

where $r=|\vec{x}|$.

In the above deducing process, we obtain an equivalent equation of (2), which is of the form (27). This fact indicates that we can do a transformation between $\mathbb{S}^{2}$ and the extend complex plane $C_{\infty}$ by (11), which is a relationship between $W$ and $S$. If we get a solution of (27), then we can transform it into the solution of (2) by (11). In the next section, the complex equation (27) will be used to construct the solution of (2), which is useful to analyze its energy property. 
Table 1 Variable separation solution, inhomogeneity terms, and decay rate of ILLG equation (2)

\begin{tabular}{ll}
\hline Solutions I-III & $q(r)$ and decay rate \\
\hline Solution I: $\left(\begin{array}{c}\cos \left(\Theta_{1}\right) \sin (B) \\
\sin \left(\Theta_{1}\right) \sin (B) \\
\cos (B)\end{array}\right)$, & $-\frac{C_{1} r^{2-2 n}\left(C_{2} r^{n}+C_{3} n\right)^{2} \cos (B) \beta^{2}}{\alpha C_{2}^{2} n^{2}}$ \\
$\Theta_{1}=-\frac{\alpha}{\cos (B) B} \ln \left(-\frac{\cos (B) \beta\left(C_{2} r^{n}+C_{3} n\right)}{\alpha n}\right)+C_{1} t$ & and $O(1)$ \\
Solution II: $\left(\begin{array}{c}\cos \left(\Theta_{2}\right) \sin (B) \\
\sin \left(\Theta_{2}\right) \sin (B) \\
\cos (B)\end{array}\right)$, & $-\frac{C_{1} \cos (B) \beta^{2}\left(\vec{r}+K_{n+1}\right)^{2}}{\alpha(\vec{k} \cdot \vec{k})}$ \\
$\Theta_{2}=C_{1} t-\frac{\alpha \ln \left(\vec{r}+K_{n+1}\right)}{\cos (B) \beta}+K_{n+2}$ & and $O(1)$ \\
\hline
\end{tabular}

Table 2 Variable separation solution, inhomogeneity terms, and decay rate of equivalent ILLG equation (27)

\begin{tabular}{ll}
\hline Solutions I-III & $q(r)$ and decay rate \\
\hline Solution I: $A \exp \left[i \Theta_{1}^{\prime}\right]$, & $\frac{C_{1} r^{-2 n+2}\left(C_{2} r^{n}+C_{3} n\right)^{2} \beta^{2}\left(A^{2}-1\right)}{\alpha\left(A^{2}+1\right) C_{2}^{2} n^{2}}$ \\
$\Theta_{1}^{\prime}=C_{1} t+\frac{\alpha\left(A^{2}+1\right)}{\beta\left(A^{2}-1\right)} \ln \left(\frac{\beta\left(A^{2}-1\right)\left(C_{2} r^{n}+C_{3} n\right)}{n \alpha\left(A^{2}+1\right)}\right)$ & and $O(1)$ \\
Solution II: $\frac{\sin (B)}{1+\cos (B)} \exp \left[i \Theta_{2}\right]$, & $-\frac{C_{1} \cos (B) \beta^{2}\left(\vec{r}+K_{n+1}\right)^{2}}{\alpha(\vec{k} \cdot \vec{k})}$ \\
$\Theta_{2}=C_{1} t-\frac{\alpha \ln \left(\vec{r}+K_{n+1}\right)}{\cos (B) \beta}+K_{n+2}$ & and $O(1)$ \\
\hline
\end{tabular}

\section{Solution of ILLG equation}

In this section, we present some solutions of ILLG equation. These solutions are all in explicit forms, which can be seen in theorems and corollaries of this section. For convenience, we list all these solutions in the Tables 1 and 2, which demonstrate the exact solutions and their decay rates.

\subsection{Solution under the cylindrical coordinates}

We construct and analyze the variable separation type solution of (4) under the cylindrical coordinates. According to (4) and (11), we use the special variable separation ansatz of the solution as follows:

$$
W=F(r) \mathrm{e}^{i M(t, r)}
$$

where the functions $F(r)$ and $M(t, r)$ (dependent variables in parentheses) are to be determined.

In the real physical model of the spin system, the inhomogeneous term of (2) and (28) are usually independent of $t$. Hence we just consider the case $q(t \vec{x})=q(r)$. This setting and (29) lead to the derivatives of time as follows:

$$
-(\alpha+\beta i) i W_{t}=(\alpha+i \beta) F M_{t} \mathrm{e}^{i M} .
$$

The structure of $q_{r} W_{r}$ is

$$
q_{r} W_{r}=q_{r} \mathrm{e}^{i M}\left(i M_{r} F+F_{r}\right)
$$

Similarly, the item associated with the Laplace and nonlinear derivatives is

$$
q\left[\Delta W-\frac{2 \bar{W} W_{r}^{2}}{1+|W|^{2}}\right]
$$




$$
\begin{aligned}
= & \frac{1}{r} q \mathrm{e}^{i M}\left(2 i F_{r} M_{r} r+i M_{r} F n+i F M_{r r} r-F M_{r}^{2} r-i M_{r} F\right. \\
& \left.+F_{r r} r+F_{r} n-F_{r}\right)-2 q F \mathrm{e}^{i M}\left(i M_{r} F+F_{r}\right)^{2} /\left(F^{2}+1\right) .
\end{aligned}
$$

Adding (30)-(32) together and multiplying with $\mathrm{e}^{-i M}$, we separate their real and imaginary parts as

$$
\left\{\begin{array}{c}
-q M_{r}^{2} F^{3} r+F^{3} M_{t} \alpha r-q F_{r} F^{2} n-q F_{r r} F^{2} r-F_{r} F^{2} q_{r} r+2 q F_{r}^{2} F r \\
\quad+q M_{r}^{2} F r+q F_{r} F^{2}+F M_{t} \alpha r-q F_{r} n-q F_{r r} r-F_{r} q_{r} r+q F_{r}=0, \\
F^{3} M_{t} \beta r-q M_{r} F^{3} n-q F^{3} M_{r r} r-M_{r} F^{3} q_{r} r+2 q F_{r} M_{r} F^{2} r+q M_{r} F^{3} \\
\quad+F M_{t} \beta r-q M_{r} F n-q F M_{r r} r-M_{r} F q_{r} r-2 q F_{r} M_{r} r+q M_{r} F=0 .
\end{array}\right.
$$

If we use $M=P(r) Q(t)$, then we obtain a blowup solution of the case $\beta=0$ of (2). However, if $\alpha \beta \neq 0$, then this special ansatzs is difficult to find out the solution of (2). In fact, we use this assumption to construct the solution of the ILLG equation, but we could not find any nontrivial solution. Hence we use another special form to find the solution of (2). We set $M$ in the variable separation form

$$
M=P(r)+Q(t) .
$$

Then (33) transforms into

$$
\left\{\begin{array}{c}
-q P_{r}^{2} F^{3} r+F^{3} Q_{t} \alpha r-q F_{r} F^{2} n-q F_{r r} F^{2} r-F_{r} F^{2} q_{r} r+2 q F_{r}^{2} F r \\
\quad+q P_{r}^{2} F r+q F_{r} F^{2}+F Q_{t} \alpha r-q F_{r} n-q F_{r r} r-F_{r} q_{r} r+q F_{r}=0, \\
F^{3} Q_{t} \beta r-q P_{r} F^{3} n-q F^{3} P_{r r} r-P_{r} F^{3} q_{r} r+2 q F_{r} P_{r} F^{2} r+q P_{r} F^{3} \\
\quad+F Q_{t} \beta r-q P_{r} F n-q F P_{r r} r-P_{r} F q_{r} r-2 q F_{r} P_{r} r+q P_{r} F=0 .
\end{array}\right.
$$

From the first and second equations of (34) we have

$$
Q=C_{1} t
$$

where $C_{1}$ is a constant.

Although $Q$ is clear from (35), it is still difficult to solve (34) under the hypothesis that $F$ is a function of $r$. However, if we set $F=A$, where $A$ is a constant, we can simplify (34) as follows:

$$
\left\{\begin{array}{l}
-q P_{r}^{2} A^{3} r+A^{3} C_{1} \alpha r+q P_{r}^{2} A r+A C_{1} \alpha r=0, \\
-A^{3} C_{1} \beta r-q P_{r} A^{3} n-q A^{3} P_{r r} r-P_{r} A^{3} q_{r} r+q P_{r} A^{3} \\
\quad+A C_{1} \beta r-q P_{r} A n-q A P_{r r} r-P_{r} A q_{r} r+q P_{r} A=0 .
\end{array}\right.
$$

From the first equation of (36) we obtain

$$
q=\frac{\alpha C_{1}\left(A^{2}+1\right)}{P_{r}^{2}\left(A^{2}-1\right)}
$$


By (37) the second equation of (36) transforms into

$$
\begin{aligned}
& -A^{4} P_{r}^{2} \beta r+A^{4} n \alpha P_{r}-A^{4} P_{r r} r \alpha-A^{4} \alpha P_{r}+2 A^{2} P_{r} n \alpha-2 A^{2} P_{r r} r \alpha \\
& -2 A^{2} P_{r} \alpha+P_{r}^{2} \beta r+P_{r} n \alpha-P_{r r} r \alpha-P_{r} \alpha=0 .
\end{aligned}
$$

Solving (38), we can find out the solution

$$
P=\frac{\alpha\left(A^{2}+1\right)}{\beta\left(A^{2}-1\right)} \ln \left(\frac{\beta\left(A^{2}-1\right)\left(C_{2} r^{n}+C_{3} n\right)}{n \alpha\left(A^{2}+1\right)}\right),
$$

where $C_{2}$ and $C_{3}$ are arbitrary constants.

Substituting (39) into (37), we obtain the exact expression of $q$.

According to the above analysis, we obtain the following:

Theorem 3.1 If the inhomogeneity term is

$$
q=\frac{C_{1} r^{-2 n+2}\left(C_{2} r^{n}+C_{3} n\right)^{2} \beta^{2}\left(A^{2}-1\right)}{\alpha\left(A^{2}+1\right) C_{2}^{2} n^{2}},
$$

then the solution of (28) is

$$
W=A \exp \left[i \frac{\alpha\left(A^{2}+1\right)}{\beta\left(A^{2}-1\right)} \ln \left(\frac{\beta\left(A^{2}-1\right)\left(C_{2} r^{n}+C_{3} n\right)}{n \alpha\left(A^{2}+1\right)}\right)+i C_{1} t\right],
$$

where $A, C_{1}, C_{2}$, and $C_{3}$ are constants.

Based on (40) and (41), we analyze the energy of this solution in the following contents. If $q=1$ and $n=2$, then the total energy of the solution of (28) is a constant due to $n=2$ is the critical case for the LLG equation. However, if the inhomogeneity term $q$ is not a constant, then ILLG may not be in critical situation. We denote by $E=\int_{\Omega} w_{E} \mathrm{~d} x$ the total energy of the solution of a partial differential equation.

The mapping target manifold of (28) is $\mathbb{S}^{2}$. Under projection, the conformal factor is

$$
g=\frac{1}{\left(1+|W|^{2}\right)^{2}}
$$

whereas the energy is

$$
\int_{0}^{\infty} q\left|W_{r}\right|^{2} g r^{n-1} \mathrm{~d} r
$$

Hence, the energy density $w_{E}$ of (41) is

$$
w_{E}(41)=\frac{\alpha C_{1} A^{2}}{A^{4}-1} \text {. }
$$

According to (11) and Theorem 3.1, we obtain the solution of (2): 
Corollary 3.2 If the inhomogeneity term is

$$
q=-\frac{C_{1} r^{2-2 n}\left(C_{2} r^{n}+C_{3} n\right)^{2} \cos (B) \beta^{2}}{\alpha C_{2}^{2} n^{2}}
$$

then the solution of $(2)$ is

$$
\left\{\begin{array}{l}
S_{1}=\cos \left(-C_{1} t+\frac{\alpha}{\cos (B) \beta} \ln \left(-\frac{\cos (B) \beta\left(C_{2} r^{n}+C_{3} n\right)}{\alpha n}\right)\right) \sin (B) \\
S_{2}=-\sin \left(-C_{1} t+\frac{\alpha}{\cos (B) \beta} \ln \left(-\frac{\cos (B) \beta\left(C_{2} r^{n}+C_{3} n\right)}{\alpha n}\right)\right) \sin (B) \\
S_{3}=\cos (B)
\end{array}\right.
$$

where $B$ is a constant, and $C_{1}, C_{2}$, and $C_{3}$ are the same constants as in Theorem 3.1.

It is clear that (44) does not tend to a trivial solution (for example, $S=(0,0,1)$ ) as $t \rightarrow+\infty$. Moreover, we cannot find out a constant value of $\lim _{t \rightarrow+\infty} S$. According to Corollary 3.2, (43) and (44) lead to

$$
\begin{aligned}
\left|S_{r}\right|^{2} & =S_{1}^{2}+S_{2}^{2}+S_{3}^{2} \\
& =-\frac{r^{2 n-2}\left((\cos (B))^{2}-1\right) \alpha^{2} n^{2} C_{2}^{2}}{\left(C_{2} r^{n}+C_{3} n\right)^{2}(\cos (B))^{2} \beta^{2}}
\end{aligned}
$$

and

$$
w_{E}(44)=q\left|S_{r}\right|^{2}=-\frac{C_{1} \alpha(\sin (B))^{2}}{\cos (B)} .
$$

According to (42) and (45), the energy density of two systems (28) and (2) are constants that are independent of time. This fact indicates that the energy of two equivalent ILLG equations tends to $+\infty$ in the whole spacial area.

\subsection{Solution under the normal coordinates}

In the last subsection, we present the exact solution under the cylindrical coordinates. We study the solution under the noncylindrical cases. The solutions obtained in this section may regard as the plane wave type solution, which is different from the cylindrical case of last subsection.

First, we search the solution of (2) under $n=3$. Denote by $x, y, z$ the different directions of space. Inspired by the last subsection, we look for the solution of (2) in the form

$$
\left\{\begin{array}{l}
S_{1}(t, x, y, z)=\cos (M(t, x, y, z)) \sin (B), \\
S_{2}(t, x, y, z)=\sin (M(t, x, y, z)) \sin (B), \\
S_{3}=\cos (B)
\end{array}\right.
$$

where $M(t, x, y, z)$ is the function to be determined. 
From (46), (5)-(10) can be simplified to

$$
\left\{\begin{array}{l}
q_{x} M_{x} \alpha+q_{y} M_{y} \alpha+q_{z} M_{z} \alpha+q M_{x x} \alpha+q M_{y y} \alpha+q M_{z z} \alpha \\
\quad+M_{y}^{2} q \cos (B) \beta+M_{z}^{2} q \cos (B) \beta+M_{x}^{2} q \cos (B) \beta=0, \\
-M_{t}-M_{z}^{2} q \cos (B) \alpha-M_{x}^{2} q \cos (B) \alpha-M_{y}^{2} q \cos (B) \alpha \\
\quad+q_{x} M_{x} \beta+q_{y} M_{y} \beta+q_{z} M_{z} \beta+q M_{x x} \beta+q M_{y y} \beta+q M_{z z} \beta=0 .
\end{array}\right.
$$

We use the ansatz of $M$ as follows:

$$
M=P(x, y, z)+Q(t) \quad \text { and } \quad Q(t)=C_{1} t
$$

where $C_{1}$ is a constant.

Substituting (48) into (47), we get

$$
\left\{\begin{array}{l}
q_{x} P_{x} \alpha+q_{y} P_{y} \alpha+q_{z} P_{z} \alpha+q P_{x x} \alpha+q P_{y y} \alpha+q P_{z z} \alpha \\
\quad+P_{y}^{2} q \cos (B) \beta+P_{z}^{2} q \cos (B) \beta+P_{x}^{2} q \cos (B) \beta=0, \\
-C_{1}-P_{z}^{2} q \cos (B) \alpha-P_{x}^{2} q \cos (B) \alpha-P_{y}^{2} q \cos (B) \alpha \\
\quad+q_{x} P_{x} \beta+q_{y} P_{y} \beta+q_{z} P_{z} \beta+q P_{x x} \beta+q P_{y y} \beta+q P_{z z} \beta=0 .
\end{array}\right.
$$

Solving (49), $P$ and $q$ are

$$
\left\{\begin{array}{l}
P=-\frac{\alpha \ln \left(x K_{1}+y K_{2}+z K_{3}+K_{4}\right)}{\cos (B) \beta}+K_{5}, \\
q=-\frac{C_{1} \cos (B) \beta^{2}\left(x K_{1}+y K_{2}+z K_{3}+K_{4}\right)^{2}}{\alpha\left(K_{1}^{2}+K_{2}^{2}+K_{3}^{2}\right)},
\end{array}\right.
$$

where $K_{i}(i=1,2,3,4,5)$ are arbitrary constants.

According to (46), (48), and (50), we obtain the solution of (2): If inhomogeneity term is

$$
q=-\frac{C_{1} \cos (B) \beta^{2}\left(x K_{1}+y K_{2}+z K_{3}+K_{4}\right)^{2}}{\alpha\left(K_{1}^{2}+K_{2}^{2}+K_{3}^{2}\right)}
$$

then the solution of (2) is

$$
\left\{\begin{array}{l}
S_{1}=\cos \left(C_{1} t-\frac{\alpha \ln \left(x K_{1}+y K_{2}+z K_{3}+K_{4}\right)}{\cos (B) \beta}+K_{5}\right) \sin (B), \\
S_{2}=\sin \left(C_{1} t-\frac{\alpha \ln \left(x K_{1}+K_{2}+z K_{3}+K_{4}\right)}{\cos (B) \beta}+K_{5}\right) \sin (B), \\
S_{3}=\cos (B)
\end{array}\right.
$$

where $K_{i}(i=1,2,3,4,5), B$, and $C_{1}$ are constants.

Expression (51) is a solution for $n=3$. Under an arbitrary integer $n$, we can find the solution of (2) similarly. We search the solution of (2) in the form

$$
\left\{\begin{array}{l}
S_{1}(t, \vec{x})=\cos \left(P(\vec{x})+C_{1} t\right) \sin (B) \\
S_{2}(t, \vec{x})=\sin \left(P(\vec{x})+C_{1} t\right) \sin (B) \\
S_{3}=\cos (B)
\end{array}\right.
$$

where $P(\vec{x})$ is the function to be determined. 
Substituting (52) into (5)-(10), we obtain

$$
\left\{\begin{array}{l}
\alpha \nabla q \cdot \nabla P+\alpha q \Delta P+\beta \cos (B) q \nabla P \cdot \nabla P=0 \\
-C_{1}-\alpha q \cos (B) \nabla P \cdot \nabla P+\beta \nabla q \cdot \nabla P+\beta q \Delta P=0 .
\end{array}\right.
$$

Solving (53), $P$ and $q$ are as follows:

$$
\left\{\begin{array}{l}
P=-\frac{\alpha \ln \left(\bar{r}+K_{n+1}\right)}{\cos (B) \beta}+K_{n+2}, \\
q=-\frac{C_{1} \cos (B) \beta^{2}\left(\vec{r}+K_{n+1}\right)^{2}}{\alpha(\vec{K} \cdot \vec{K})}
\end{array}\right.
$$

where $K_{i}(i=1,2,3, \ldots)$ are arbitrary constants, $\vec{K}=\left(K_{1}, K_{2}, K_{3}, \ldots, K_{n}\right)$, and $\vec{r}=\vec{K} \cdot \vec{x}$. According to (52) and (54), we obtain the solution of (2) as follows.

Theorem 3.3 If the inhomogeneity term is

$$
q=-\frac{C_{1} \cos (B) \beta^{2}\left(\vec{r}+K_{n+1}\right)^{2}}{\alpha(\vec{K} \cdot \vec{K})},
$$

then the solution of (2) is

$$
\left\{\begin{array}{l}
S_{1}=\cos \left(C_{1} t-\frac{\alpha \ln \left(\bar{r}+K_{n+1}\right)}{\cos (B) \beta}+K_{n+2}\right) \sin (B) \\
S_{2}=\sin \left(C_{1} t-\frac{\alpha \ln \left(\bar{r}+K_{n+1}\right)}{\cos (B) \beta}+K_{n+2}\right) \sin (B) \\
S_{3}=\cos (B)
\end{array}\right.
$$

Similarly to (44), (56) does not tend to a trivial solution as $t \rightarrow+\infty$ too. Moreover, $\lim _{t \rightarrow+\infty} S$ does not exist. It is easy to find the solution of (27). Employing (56) and (11), we obtain the following:

Corollary 3.4 If the inhomogeneity term satisfies (55), then the solution of (27) is

$$
W=\frac{\sin (B)}{1+\cos (B)} \exp \left[i\left(C_{1} t-\frac{\alpha \ln \left(\bar{r}+K_{n+1}\right)}{\cos (B) \beta}+K_{n+2}\right)\right]
$$

The energy density of the solutions presented in Theorem 3.3 and Corollary 3.4 shows a similar property as the solutions of the previous subsection. According to Corollary 3.4, we obtain

$$
|\nabla S|^{2}=\frac{(\sin (B))^{2} \alpha^{2}(|\vec{K}|)}{\left(\vec{r}+K_{n+1}\right)^{2}(\cos (B))^{2} \beta^{2}}
$$

and

$$
w_{E}(56)=q|\nabla S|^{2}=-\frac{C_{1} \alpha(\sin (B))^{2}}{\cos (B)} .
$$


Similarly to (45), the energy density (58) is constant. This situation will be the same for (41) and (57):

$$
w_{E}(57)=q \frac{|\nabla W|^{2}}{\left(1+|W|^{2}\right)^{2}}=-\frac{C_{1} \alpha(\sin (B))^{2}(\cos (B)+1)^{2}}{4 \cos (B)} .
$$

In this section, we present two solutions of (2) (or (27)). For convenience, we summarize some information about these solutions in Tables 1 and 2, respectively. From these tables we can see the exact form of variable separation solution, inhomogeneity terms, and decay rates. According to the solutions of the ILLG equation in the tables, we can see that $\alpha$ and $\beta$ cannot be 0 since the denominator cannot be 0 . Although the solutions of the ILLG equation does not degenerate into the trivial solutions as $t \rightarrow+\infty$, their decay rates are all independent of the spatial variable $\vec{x}$. More exactly, the decay rate of the solutions for the ILLG equation are $O(1)$. Different from the solutions presented in our previous work $[28,29]$, the solutions in Tables 1 and 2 do not develop any blowup at any time.

\section{Solution of Schrödinger map equation}

In this section, we study the solution of the LLG equation without damping. More clearly, we set $q=1$ and $\beta=0$ in (2). In this setting, (2) degenerates into the simple form

$$
\frac{\partial}{\partial t} S=S \Lambda \Delta S
$$

Therefore $W$ satisfies

$$
-i W_{t}=\Delta W-\frac{2 \bar{W}}{1+|W|^{2}}\langle\nabla W\rangle
$$

Although (59) is simpler than (2), the solution is very rare as far as we see. For the details of the exact solution of (59), we refer the reader to [9, 12, 27].

\subsection{Solution under the cylindrical coordinates}

In the last section, we obtain some variable separation solutions such as (44) $(q$ as (43)) and (56) ( $q$ as (55)). According to these solutions, we can see that the extreme case $\alpha=0$ and $\beta=0$ are not included since the denominator is not zero. If we settle down on the variable separation form provided in the last section, what will be the solution like? Motivated by this, we consider the solution

$$
\left\{\begin{array}{l}
S_{1}(t, r)=\cos (N(t, r)) \sin (G(r)) \\
S_{2}(t, r)=\sin (N(t, r)) \sin (G(r)) \\
S_{3}(r)=\cos (G(r))
\end{array}\right.
$$

where $N(t, r)$ and $G(r)$ are functions to be determined.

We consider the case where $q(t \vec{x})$ does not contain $t$. Then, by (61), (2) is greatly simplified and transforms into

$$
-\sin (G) N_{r}+2 \cos (G) G_{r} N_{r} r+\sin (G) N_{r} n+\sin (G) N_{r r} r=0
$$


and

$$
\sin (G) \cos (G) N_{r}^{2} r-G_{r} n-G_{r r} r+\sin (G) N_{t} r+G_{r}=0
$$

which are nonlinear partial differential equations. To solve these equations, let us assume that $N$ is a variable separation function,

$$
N=M_{1}(r)+M_{2}(t)
$$

where $M_{1}(r)$ and $M_{2}(t)$ depend on $r$ and $t$, respectively. Substituting (64) into (62) and (63), we obtain

$$
\begin{aligned}
M_{2}= & C_{5} t, \\
& -\sin (G) M_{1 r}+2 \cos (G) G_{r} M_{1 r} r+\sin (G) M_{1 r} n+\sin (G) M_{1 r r} r=0,
\end{aligned}
$$

and

$$
\sin (G) \cos (G) M_{1 r}^{2} r-G_{r} n-G_{r r} r+\sin (G) C_{5} r+G_{r}=0 .
$$

Equations (65)-(66) are ordinary differential equations (ODEs), which are hard to solve explicitly. However, we can find out some constraints of the solution that are useful for analysis of the decay rate of the energy density. If we use

$$
G=2 \arctan \left(\mathrm{e}^{F}\right)
$$

then (65)-(66) transform into

$$
\begin{gathered}
2 M_{1 r} F_{r} \mathrm{e}^{2 F} r-M_{1 r} \mathrm{e}^{2 F} n-M_{1 r r} \mathrm{e}^{2 F} r-2 M_{1 r} r F_{r} \\
+M_{1 r} \mathrm{e}^{2 F}-M_{1 r} n-M_{1 r r} r+M_{1 r}=0
\end{gathered}
$$

and

$$
\begin{aligned}
& M_{1 r}^{2} \mathrm{e}^{2 F} r-F_{r}^{2} \mathrm{e}^{2 F} r+F_{r r} \mathrm{e}^{2 F} r+F_{r} \mathrm{e}^{2 F} n-C_{5} \mathrm{e}^{2 F} r-M_{1 r}^{2} r \\
& +r F_{r}^{2}-F_{r} \mathrm{e}^{2 F}+r F_{r r}+n F_{r}-C_{5} r-F_{r}=0 .
\end{aligned}
$$

From (68) we obtain

$$
M_{1}=C_{1}+C_{2} \int r^{-n+1}\left(\mathrm{e}^{4 F}+2 \mathrm{e}^{2 F}+1\right) \mathrm{e}^{-2 F} \mathrm{~d} r .
$$

Substituting (70) into (69), we have

$$
\begin{aligned}
& 3 r^{-2 n+3} \mathrm{e}^{4 F} C_{2}^{2}+2 r^{-2 n+3} \mathrm{e}^{2 F} C_{2}^{2}+r^{-2 n+3} \mathrm{e}^{6 F} C_{2}^{2}-2 r^{-2 n+3} C_{2}^{2} \\
& -3 r^{-2 n+3} \mathrm{e}^{-2 F} C_{2}^{2}-r^{-2 n+3} \mathrm{e}^{-4 F} C_{2}^{2}-F_{r}^{2} \mathrm{e}^{2 F} r+\mathrm{e}^{2 F} F_{r r} r+F_{r} \mathrm{e}^{2 F} n \\
& -\mathrm{e}^{2 F} r C_{5}+r F_{r}^{2}+r F_{r r}-F_{r} \mathrm{e}^{2 F}+n F_{r}-C_{5} r-F_{r}=0 .
\end{aligned}
$$


We cannot find the solution of (71). However, there is some additional information about $F$. If we solve (34), then we can find

$$
M_{1}=\int \frac{C_{3}^{2} r^{-n+1}}{(\sin (G))^{2}} \mathrm{~d} r+C_{4}
$$

If we set $C_{4}=C_{1}$ and $C_{2}=1 /\left(2 C_{3}\right)^{2}$, then (72) and (70) will be of the same form. Substituting (72) into (66), we obtain

$$
G_{r r}+\frac{(n-1) G_{r}}{r}-\frac{\cos (G) C_{3}^{4} r^{-2 n+2}}{(\sin (G))^{3}}-\sin (G) C_{5}=0 .
$$

Equation (73) is just an ODE, which is like a sine-Gordon equation. Because (73) is not a standard sine-Gordon equation, its existence details are not so clear. In fact, we rewrite (73) as

$$
G_{r r}=f\left(r, G, G_{r}\right)
$$

where

$$
f\left(r, G, G_{r}\right)=-\frac{(n-1) G_{r}}{r}+\frac{\cos (G) C_{3}^{4} r^{-2 n+2}}{(\sin (G))^{3}}+\sin (G) C_{5} .
$$

We consider the Cauchy problem of (74): finding a solution of equation (74) satisfying the initial conditions

$$
G(\varepsilon)=G_{\varepsilon} \quad \text { and } \quad G_{r}(\varepsilon)=G_{r \varepsilon} \quad\left(0<\varepsilon<+\infty, 0<G_{\varepsilon}<\pi\right) .
$$

Obviously, $f\left(r, G, G_{r}\right)$ is a continuous function of all its arguments in a neighborhood of a point $\left(\varepsilon, G_{\varepsilon}, G_{r \varepsilon}\right)$. Furthermore, it is not difficult to verify that $f\left(r, G, G_{r}\right)$ have bounded partial derivatives $\frac{\partial f}{\partial G}$ and $\frac{\partial f}{\partial G_{r}}$ in this neighborhood. Hence, a solution of (74) (or (73)) satisfying the initial conditions (75) exists and is unique. How to construct a reasonable initial condition that satisfies (74)? We just need to employ the setting (67).

Due to (72), if we obtain the solution of (73), then we can find the solution of (71). According to the above results, we obtain the following:

Theorem 4.1 Let F be a function satisfying (71). Tthen the solution of (59) is

$$
\left\{\begin{array}{l}
S_{1}(t, r)=\frac{2 \mathrm{e}^{F}}{1+\mathrm{e}^{2 F}} \cos \left(C_{1}+C_{2} \int r^{-n+1}\left(\mathrm{e}^{2 F}+1\right)^{2} \mathrm{e}^{-2 F} \mathrm{~d} r+C_{5} t\right), \\
S_{2}(t, r)=\frac{2 \mathrm{e}^{F}}{1+\mathrm{e}^{2 F}} \sin \left(C_{1}+C_{2} \int r^{-n+1}\left(\mathrm{e}^{2 F}+1\right)^{2} \mathrm{e}^{-2 F} \mathrm{~d} r+C_{5} t\right), \\
S_{3}(r)=\frac{1-\mathrm{e}^{2 F}}{1+\mathrm{e}^{2 F}},
\end{array}\right.
$$

whereas

$$
W=\mathrm{e}^{F} \exp \left[i\left(C_{1}+C_{2} \int r^{-n+1}\left(\mathrm{e}^{2 F}+1\right)^{2} \mathrm{e}^{-2 F} \mathrm{~d} r+C_{5} t\right)\right]
$$

is a solution of $(60)$. 
Although we cannot solve (71), we can deduce some its scale as $r \rightarrow+\infty$. Substituting $F=K_{1} \ln (K r)(K>0)$ into the left side of $(71)$, we obtain

$$
\begin{aligned}
3 r^{-2 n+4+4 K_{1}} K^{4 K_{1}} C_{2}^{2}+2 r^{-2 n+4+2 K_{1}} K^{2 K_{1}} C_{2}^{2}+r^{-2 n+4+6 K_{1}} K^{6 K_{1}} C_{2}^{2} \\
\quad-2 r^{-2 n+4} C_{2}^{2}-3 r^{-2 n+4-2 K_{1}} K^{-2 K_{1}} C_{2}^{2}-r^{-2 n+4-4 K_{1}} K^{-4 K_{1}} C_{2}^{2} \\
-K_{1}^{2} K^{2 K_{1}} r^{2 K_{1}}-2 K^{2 K_{1}} r^{2 K_{1}} K_{1}+K_{1} K^{2 K_{1}} r^{2 K_{1}} n-K^{2 K_{1}} r^{2 K_{1}+2} C_{5}+K_{1}^{2} \\
\quad-2 K_{1}+n K_{1}-C_{5} r^{2} \triangleq H(r) .
\end{aligned}
$$

If $C_{5}>0$ and $n \geq 2$, then the leading-order term of (78) is

$$
r^{-2 n+4+6 K_{1}} K^{6 K_{1}} C_{2}^{2}-K^{2 K_{1}} r^{2 K_{1}+2} C_{5} \triangleq L_{C_{5}>0},
$$

and we obtain

$$
K_{1}=\frac{n}{2}-\frac{1}{2}
$$

So, as $r \rightarrow+\infty$, we have

$$
\lim _{r \rightarrow+\infty} H(r)=\operatorname{sign}\left[K^{n-1}\left(K^{2 n-2} C_{2}^{2}-C_{5}\right)\right] \cdot \infty .
$$

Relationship (79) means that $K$ determines the limit to be a positive or negative value of $H(r)$ for sufficiently large $r$. In this situation, we have

$$
\left(\frac{n}{2}-\frac{1}{2}\right) \ln \left(K_{A} r\right)<F<\left(\frac{n}{2}-\frac{1}{2}\right) \ln \left(K_{B} r\right) \quad\left(0<K_{A}<K_{B}\right) .
$$

Similarly, if $C_{5}<0$ and $n \geqq 2$, then the leading-order term of (78) is

$$
-r^{-2 n+4-4 K_{1}} K^{-4 K_{1}} C_{2}^{2}-C_{5} r^{2} \triangleq L_{C_{5}<0},
$$

where

$$
K_{1}=-\frac{n}{2}+\frac{1}{2}
$$

In this case, we obtain

$$
\lim _{r \rightarrow+\infty} H(r)=-\operatorname{sign}\left(K^{2 n-2} C_{2}^{2}+C_{5}\right) \cdot \infty .
$$

If $r$ is sufficiently large, then (81) indicates that

$$
\left(-\frac{n}{2}+\frac{1}{2}\right) \ln \left(K_{C} r\right)<F<\left(-\frac{n}{2}+\frac{1}{2}\right) \ln \left(K_{D} r\right) \quad\left(0<K_{C}<K_{D}\right) .
$$

If $n \geq 2$, then by (80) and (82) we have (for sufficiently large $r$ )

$$
\left(\frac{n}{2}-\frac{1}{2}\right) \ln \left(K_{E} r\right)<|F|<\left(\frac{n}{2}-\frac{1}{2}\right) \ln \left(K_{F} r\right) \quad\left(0<K_{E}<K_{F}\right) .
$$


From (61), (64), (67), and (70) we have

$$
\begin{aligned}
w_{E}(76) & =S_{1 r}^{2}+S_{2 r}^{2}+S_{3 r}^{2} \\
& =N_{r}^{2}(\sin (G))^{2}+G_{r}^{2} \\
& =M_{1 r}^{2}(\sin (G))^{2}+G_{r}^{2} \\
& =\frac{C_{3}^{4} r^{-2 n+2}}{(\sin (G(r)))^{2}}+G_{r}^{2} \\
& =\frac{4 r^{2-2 n} F^{2} C_{2}^{2}\left(\mathrm{e}^{2 F}+4\right)\left(\mathrm{e}^{2 F}+\mathrm{e}^{-2 F}\right)}{\left(F^{2}+1\right)^{2}}+\frac{24 r^{2-2 n} F^{2} C_{2}^{2}+4 F_{r}^{2}}{\left(F^{2}+1\right)^{2}} .
\end{aligned}
$$

Substituting

$$
F= \pm\left(\frac{n}{2}-\frac{1}{2}\right) \ln (K r)
$$

into (83), we obtain

$$
\begin{aligned}
w_{E}(76)= & 16 K^{-2} r^{-2}(-1+n)^{2}(\ln (K r))^{2}\left(r^{-4 n+6} K^{-2 n+4} C_{2}^{2}\right. \\
& +4 r^{-3 n+5} K^{-n+3} C_{2}^{2}+6 r^{-2 n+4} C_{2}^{2} K^{2}+4 r^{-n+3} K^{n+1} C_{2}^{2} \\
& \left.+r^{2} K^{2 n} C_{2}^{2}+K^{2}\right) /(\ln (K r))^{2} n^{2}-2(\ln (K r))^{2} n \\
& \left.+(\ln (K r))^{2}+4\right)^{2} .
\end{aligned}
$$

According to (85), the scale of the energy density is

$$
w_{E}(76) \sim O(1) \quad(n \geq 2)
$$

as $r \rightarrow+\infty$.

The energy density of (77) is

$$
w_{E}(77)=\frac{\left|W_{r}\right|^{2}}{\left(1+|W|^{2}\right)^{2}}=\frac{\mathrm{e}^{2 F}\left(\left(r^{-n+1}\right)^{2}\left(\mathrm{e}^{4 F}+2 \mathrm{e}^{2 F}+1\right)^{2}\left(\mathrm{e}^{-2 F}\right)^{2} C_{2}^{2}+F_{r}^{2}\right)}{\left(1+\mathrm{e}^{2 F}\right)^{2}}
$$

Employing (84), the right side of (86) changes into

$$
\begin{aligned}
3 r^{-2 n+4+4 K_{1}} K^{4 K_{1}} C_{2}^{2}+2 r^{-2 n+4+2 K_{1}} K^{2 K_{1}} C_{2}^{2}+r^{-2 n+4+6 K_{1}} K^{6 K_{1}} C_{2}^{2} \\
-2 r^{-2 n+4} C_{2}^{2}-3 r^{-2 n+4-2 K_{1}} K^{-2 K_{1}} C_{2}^{2}-r^{-2 n+4-4 K_{1}} K^{-4 K_{1}} C_{2}^{2} \\
-K_{1}^{2} K^{2 K_{1}} r^{2 K_{1}}-2 K^{2 K_{1}} r^{2 K_{1}} K_{1}+K_{1} K^{2 K_{1}} r^{2 K_{1}} n-K^{2 K_{1}} r^{2 K_{1}+2} C_{5}+K_{1}^{2} \\
-2 K_{1}+n K_{1}-C_{5} r^{2} \triangleq H(r) .
\end{aligned}
$$

If $C_{5}>0$ and $n \geq 2$, then the leading-order term of (87) is

$$
r^{-2 n+4+6 K_{1}} K^{6 K_{1}} C_{2}^{2}-K^{2 K_{1}} r^{2 K_{1}+2} C_{5} \triangleq L_{C_{5}>0},
$$


and we obtain

$$
K_{1}=\frac{n}{2}-\frac{1}{2}
$$

So, as $r \rightarrow+\infty$, we have

$$
\lim _{r \rightarrow+\infty} H(r)=\operatorname{sign}\left[K^{n-1}\left(K^{2 n-2} C_{2}^{2}-C_{5}\right)\right] \cdot \infty
$$

and

$$
\begin{aligned}
w_{E}(77)= & (4 K r)^{-1}\left(4 K^{-n+4} r^{-3 n+6} C_{2}^{2}+16 r^{5-2 n} K^{3} C_{2}^{2}\right. \\
& +24 K^{n+2} r^{-n+4} C_{2}^{2}+16 K^{2 n+1} r^{3} C_{2}^{2}+K^{n+2} r^{n} n^{2} \\
& \left.+4 K^{3 n} r^{n+2} C_{2}^{2}-2 K^{n+2} r^{n} n+K^{n+2} r^{n}\right) /\left(K^{n} r^{n}+K r\right)^{2} .
\end{aligned}
$$

Relation (88) shows that the scale of the energy density is

$$
w_{E}(77) \sim \frac{1}{r^{n-1}} \quad(n \geq 2)
$$

as $r \rightarrow+\infty$.

If $n=1$, then $F$ presents a different behavior as $r \rightarrow+\infty$. Exactly, $F$ is not a $K_{1} \ln (K r)$ scale, but

$$
F \sim \ln (K \arctan (r))
$$

as $r \rightarrow+\infty$.

Substituting $n=1$ and $F=K \ln (\arctan (r))$ into the left side of (71), we obtain

$$
\begin{aligned}
& r^{2} K^{6}(\arctan (r))^{6} C_{2}^{2}-\frac{r^{2} C_{2}^{2}}{K^{4}(\arctan (r))^{4}}+3 r^{2} K^{4}(\arctan (r))^{4} C_{2}^{2}-\frac{3 r^{2} C_{2}^{2}}{K^{2}(\arctan (r))^{2}} \\
& -\frac{2 K^{2} r^{2}}{\left(r^{2}+1\right)^{2}}+2 r^{2} K^{2}(\arctan (r))^{2} C_{2}^{2}-\frac{2 r^{3} K^{2} \arctan (r)}{\left(r^{2}+1\right)^{2}}-r^{2} K^{2}(\arctan (r))^{2} C_{5} \\
& -2 r^{2} C_{2}^{2}-\frac{2 r^{3}}{\left(r^{2}+1\right)^{2} \arctan (r)}-C_{5} r^{2} \triangleq H_{1}(r) .
\end{aligned}
$$

According to (90), its leading-order term is

$$
\begin{gathered}
r^{2} K^{6}(\arctan (r))^{6} C_{2}^{2}-\frac{r^{2} C_{2}^{2}}{K^{4}(\arctan (r))^{4}}+3 r^{2} K^{4}(\arctan (r))^{4} C_{2}^{2}-\frac{3 r^{2} C_{2}^{2}}{K^{2}(\arctan (r))^{2}} \\
+2 r^{2} K^{2}(\arctan (r))^{2} C_{2}^{2}-r^{2} K^{2}(\arctan (r))^{2} C_{5}-2 r^{2} C_{2}^{2}-C_{5} r^{2} \triangleq H_{2}(r) .
\end{gathered}
$$

As $r \rightarrow+\infty, \arctan (r) \rightarrow \pi / 2$. Substituting $\arctan (r)=\pi / 2$ into (91), $H_{2}(r)$ becomes

$$
\begin{aligned}
& \frac{r^{2} K^{6} \pi^{6} C_{2}^{2}}{64}-\frac{16 r^{2} C_{2}^{2}}{K^{4} \pi^{4}}+\frac{3}{16} r^{2} K^{4} \pi^{4} C_{2}{ }^{2}-\frac{12 r^{2} C_{2}^{2}}{K^{2} \pi^{2}} \\
& +\frac{1}{2} r^{2} K^{2} \pi^{2} C_{2}^{2}-\frac{1}{4} r^{2} K^{2} \pi^{2} C_{5}-2 r^{2} C_{2}^{2}-C_{5} r^{2} \triangleq H_{3}(r) .
\end{aligned}
$$


If $C_{5} \neq 0$, then, as $r \rightarrow+\infty$, we have

$$
\begin{aligned}
\lim _{r \rightarrow+\infty} H_{3}(r)= & \operatorname{sign}\left[K^{8} \pi^{8} C_{2}^{2}+8 K^{6} \pi^{6} C_{2}^{2}-16 K^{4} \pi^{4} C_{5}\right. \\
& \left.-128 K^{2} \pi^{2} C_{2}^{2}-256 C_{2}^{2}\right] \cdot \infty
\end{aligned}
$$

where $K>0$.

For sufficiently large $r$, (92) means that $K>0$ determines the limit to be a positive or negative value of $H_{3}(r)$ According to (92), we prove (89). The decay rate of energy density is clear due to (89) under $n=1$. So, we obtain (here we set $A_{r}=\arctan (r)$ )

$$
\begin{aligned}
w_{E}(76) \sim & 4\left(\left(\ln \left(K A_{r}\right)\right)^{2} K^{8} A_{r}{ }^{8} C_{2}^{2}+4\left(\ln \left(K A_{r}\right)\right)^{2} K^{6} A_{r}{ }^{6} C_{2}^{2}\right. \\
& +6\left(\ln \left(K A_{r}\right)\right)^{2} K^{4} A_{r}{ }^{4} C_{2}^{2}+\frac{A R^{2} K^{4}}{\left(r^{2}+1\right)^{2}}+4\left(\ln \left(K A_{r}\right)\right)^{2} K^{2} A_{r}{ }^{2} C_{2}^{2} \\
& \left.+\left(\ln \left(K A_{r}\right)\right)^{2} C_{2}^{2}\right) /\left[\left(1+\left(\ln \left(K A_{r}\right)\right)^{2}\right)^{2} K^{4} A_{r}{ }^{4}\right] \\
\sim & O(1)
\end{aligned}
$$

as $r \rightarrow+\infty$.

Similarly, the energy density of (77) under $n=1$ is

$$
\begin{aligned}
w_{E}(77) \sim & \left(K^{6} A_{r}^{6} C_{2}^{2}+4 K^{4} A_{r}^{4} C_{2}^{2}+\frac{K^{2}}{\left(r^{2}+1\right)^{2}}+6 K^{2} A_{r}^{2} C_{2}^{2}\right. \\
& \left.+4 C_{2}^{2}+\frac{C_{2}^{2}}{K^{2} A_{r}^{2}}\right) /\left(K^{2} A_{r}^{2}+1\right)^{2} \sim O(1)
\end{aligned}
$$

as $r \rightarrow+\infty$.

From the above analysis, we can conclude that: if $F$ satisfies (71), then we have

Theorem 4.2 If $n \geq 2$, then the energy densities of (76) and (77) are

$$
w_{E}(76) \sim O(1) \quad \text { and } \quad w_{E}(77) \sim \frac{1}{r^{n-1}}
$$

as $r \rightarrow+\infty$, respectively, whereas

$$
w_{E}((76) \text { or }(77)) \sim O(1) \quad(n=1)
$$

\subsection{Solution under the normal coordinates}

Under the arbitrary integer $n$, we can similarly find the solution of (59). We search the solution of (59) of the form

$$
\left\{\begin{array}{l}
S_{1}(t, \vec{x})=\cos \left(M_{2}(\vec{x})+C_{5} t\right) \sin \left(G_{2}(\vec{x})\right), \\
S_{2}(t, \vec{x})=\sin \left(M_{2}(\vec{x})+C_{5} t\right) \sin \left(G_{2}(\vec{x})\right), \\
S_{3}(\vec{x})=\cos \left(G_{2}(\vec{x})\right)
\end{array}\right.
$$

where $M_{2}(\vec{x})$ and $G_{2}(\vec{x})$ are functions to be determined. 
Substituting (93) into (59), we obtain

$$
\left\{\begin{array}{l}
2 \cos \left(G_{2}\right) \nabla G_{2} \cdot \nabla M_{2}+\sin G_{2} \Delta M_{2}=0 \\
\sin \left(G_{2}\right) \cos \left(G_{2}\right) \nabla M_{2} \cdot \nabla M_{2}+C_{5} \sin \left(G_{2}\right)-\Delta G_{2}=0
\end{array}\right.
$$

We study the plane wave solution of (94). Here we set $L_{i}(i=1,2,3, \ldots)$ as arbitrary constants, $\vec{L}=\left(L_{1}, L_{2}, L_{3}, \ldots, L_{n}\right)$, and $\vec{r}=\vec{L} \cdot \vec{x}$. So, (94) transforms into

$$
\left\{\begin{array}{l}
2 \cos \left(G_{2}\right) G_{2 \bar{r}} M_{2 \bar{r}}+\sin G_{2} M_{2 \overline{r r}}=0, \\
|\vec{L}|^{2} \sin (G) \cos (G) M_{2 \bar{r}}^{2}+C_{5} \sin (G)-|\vec{L}|^{2} G_{2 \overline{r r}}=0 .
\end{array}\right.
$$

Equation (95) is a nonlinear ODE. Solving the first equation of (95), we obtain

$$
M_{2}=C_{1}+C_{2} \int\left(\sin \left(G_{2}\right)\right)^{-2} \mathrm{~d} \bar{r}
$$

Employing (96), the second equation of (95) transforms into

$$
G_{2 r r}-\sin \left(G_{2}\right) C_{5}-\frac{1}{\left(\sin \left(G_{2}\right)\right)^{3}}\left(\frac{C_{5}}{|\vec{L}|^{2}}-C_{5}+\cos \left(G_{2}\right) C_{2}^{2}\right)=0
$$

where

$$
L_{C}=\frac{C_{5}}{|\vec{L}|^{2}}-C_{5}
$$

If $G_{2}$ satisfies (97) $\left(M_{2}\right.$ satisfies (96)), then we have

$$
\begin{aligned}
w_{E}(93) & =|\vec{L}|^{2}\left(S_{1 \bar{r}}^{2}+S_{2 \bar{r}}^{2}+S_{3 \bar{r}}^{2}\right) \\
& =|\vec{L}|^{2}\left(M_{2 \bar{r}}^{2}\left(\sin \left(G_{2}\right)\right)^{2}+G_{2 \bar{r}}^{2}\right) \\
& =|\vec{L}|^{2}\left(G_{2 r}^{2}+\frac{C_{2}^{2}}{\left(\sin \left(G_{2}\right)\right)^{2}}\right)
\end{aligned}
$$

Equation (98) is similar to (73), whereas (96) is similar to (70) under $n=1$. We also use the transformation like (67)

$$
G_{2}=2 \arctan \left(\mathrm{e}^{F_{2}}\right),
$$

which transforms (97) into

$$
\begin{aligned}
2 \mathrm{e}^{F_{2}} C_{2}^{2}+\mathrm{e}^{-3 F_{2}} C_{2}^{2}+3 \mathrm{e}^{-F_{2}} C_{2}^{2}-2 \mathrm{e}^{3 F_{2}} C_{2}^{2}-16 \mathrm{e}^{F_{2}} F_{2 r}^{2}-16 \mathrm{e}^{F_{2}} F_{2 r r} \\
\quad+16 \mathrm{e}^{F_{2}} C_{5}-\mathrm{e}^{7 F_{2}} C_{2}^{2}-3 \mathrm{e}^{5 F_{2}} C_{2}^{2}+16 \mathrm{e}^{3 F_{2}} F_{r}^{2}-16 \mathrm{e}^{3 F_{2}} F_{2 r r}+16 \mathrm{e}^{3 F_{2}} C_{5} \\
\quad+L_{C}\left(\mathrm{e}^{7 F_{2}}+5 \mathrm{e}^{5 F_{2}}+10 \mathrm{e}^{3 F_{2}}+\mathrm{e}^{-3 F_{2}}+10 \mathrm{e}^{F_{2}}+5 \mathrm{e}^{-F_{2}}\right)=0 .
\end{aligned}
$$


For simplicity, we just consider the case of $C_{2}^{2}>L_{C}$. Substituting $F_{2}=\ln (K \arctan (\bar{r}))(K>$ 0 ) into (99) and then extracting the coefficient of the highest order of $\bar{r}$, we obtain the coefficient of $\bar{r}^{4}$ (setting $A_{\bar{r}}=\arctan (\bar{r})$ ),

$$
\begin{aligned}
& \left(A_{\bar{r}}^{10} C_{2}^{2}-A_{\bar{r}}^{10} L_{C}\right) K^{10}+\left(2 A_{\bar{r}}^{6} C_{2}^{2}-16 A_{\bar{r}}^{6} C_{5}-10 A_{\bar{r}}^{6} L_{C}\right) K^{6} \\
& \quad+\left(3 A_{\bar{r}}^{8} C_{2}^{2}-5 A_{\bar{r}}^{8} L_{C}\right) K^{8}+\left(-2 A_{r}^{4} C_{2}^{2}-16 A_{\bar{r}}^{4} C_{5}-10 A_{\bar{r}}^{4} L_{C}\right) K^{4} \\
& \quad+\left(-3 A_{\bar{r}}^{2} C_{2}^{2}-5 A_{\bar{r}}^{2} L_{C}\right) K^{2}-C_{2}^{2}-L_{C} \triangleq H_{4}(K)
\end{aligned}
$$

The term $H_{4}(K) \bar{r}^{4}$ is the leading-order term as $\bar{r} \rightarrow+\infty$. We can control the positive and negative values of $H_{4}(K)$ by $K$. Hence, if $C_{2}^{2}>L_{C}$, then we obtain

$$
F_{2} \sim \ln (K \arctan (\bar{r})) \quad(K>0)
$$

as $\bar{r} \rightarrow+\infty$.

Employing (98) and (100), it is simple to check that

$$
w_{E}(93)=\frac{4|\vec{L}|^{2} F_{2 \bar{r}}^{2}}{\left(F_{2}^{2}+1\right)^{2}}+\frac{|\vec{L}|^{2} C_{2}^{2}\left(F_{2}^{2}+1\right)^{2}}{4 F_{2}^{2}} \sim O(1)
$$

as $\bar{r} \rightarrow+\infty$.

Similarly to the proof of Theorem 4.2, there exists a function $F$ satisfying (99). In this setting, we obtain the following:

Theorem 4.3 Suppose there exists a function $F_{2}$ satisfying (99). Then the solution of (59) is

$$
\left\{\begin{array}{l}
S_{1}(t, \bar{r})=\frac{2 \mathrm{e}_{2}}{1+\mathrm{e}^{2 F_{2}}} \cos \left(C_{1}+C_{2} \int\left(\mathrm{e}^{2 F_{2}}+1\right)^{2} \mathrm{e}^{-2 F_{2}} \mathrm{~d} \bar{r}+C_{5} t\right) \\
S_{2}(t, \bar{r})=\frac{2 \mathrm{e}_{2}}{1+\mathrm{e}^{2 F_{2}}} \sin \left(C_{1}+C_{2} \int\left(\mathrm{e}^{2 F_{2}}+1\right)^{2} \mathrm{e}^{-2 F_{2}} \mathrm{~d} \bar{r}+C_{5} t\right) \\
S_{3}(\bar{r})=\frac{1-\mathrm{e}^{2 F_{2}}}{1+\mathrm{e}^{2 F_{2}}}
\end{array}\right.
$$

whereas

$$
W=\mathrm{e}^{F_{2}} \exp \left[i\left(C_{1}+C_{2} \int\left(\mathrm{e}^{2 F_{2}}+1\right)^{2} \mathrm{e}^{-2 F_{2}} \mathrm{~d} \bar{r}+C_{5} t\right)\right]
$$

is a solution of $(60)$.

Furthermore, if $C_{2}^{2}>L_{C}$, then the decay rate of energy density is

$$
w_{E}((101) \text { or }(102)) \sim O(1)
$$

as $\bar{r} \rightarrow+\infty$.

In Tables 3 and 4, we present the variable separation solution (and energy density) of (59) and (60), respectively. Similarly to Tables 1 and 2, most of the decay rates of the solutions are in a scale of $O(1)$ in Tables 3 and 4. The only nonconstant decay rate of the solution is the solution I in Table 3. Its decay rate is related to the dimension $n$ as $1 / r^{n-1}$. If $n=1$, then the decay rate degenerates to the constant scale $O(1)$. Similarly to the solutions in Tables 1 and 2, the solutions in Tables 3 and 4 develop no any singularity. 
Table 3 Variable separation solution of the LLG equation without damping (see equation (59)). $F$ is the solution of (71); $F_{2}$ satisfies (99)

\begin{tabular}{|c|c|c|}
\hline \multicolumn{2}{|c|}{ Solutions I-II } & Decay rate \\
\hline Solution I: & $\left.\begin{array}{c}\frac{2 \mathrm{e}^{F}}{1+\mathrm{e}^{2 F}} \cos \left(C_{1}+C_{2} \int r^{-n+1}\left(\mathrm{e}^{2 F}+1\right)^{2} \mathrm{e}^{-2 F} \mathrm{~d} r+C_{5} t\right) \\
\frac{2 \mathrm{e}^{F}}{1+\mathrm{e}^{2 F}} \sin \left(C_{1}+C_{2} \int r^{-n+1}\left(\mathrm{e}^{2 F}+1\right)^{2} \mathrm{e}^{-2 F} \mathrm{~d} r+C_{5} t\right) \\
\frac{1-\mathrm{e}^{2 F}}{1+\mathrm{e}^{2 F}}\end{array}\right)$ & $O(1)$ \\
\hline Solution II: & $\left(\begin{array}{c}\frac{2 \mathrm{e}_{2}}{1+\mathrm{e}^{2 F_{2}}} \cos \left(C_{1}+C_{2} \int\left(\mathrm{e}^{2 F_{2}}+1\right)^{2} \mathrm{e}^{-2 F_{2}} \mathrm{~d} \bar{r}+C_{5} t\right) \\
\frac{2 \mathrm{e}_{2}}{1+\mathrm{e}^{2 F_{2}}} \sin \left(C_{1}+C_{2} \int\left(\mathrm{e}^{2 F_{2}}+1\right)^{2} \mathrm{e}^{-2 F_{2}} \mathrm{~d} \bar{r}+C_{5} t\right) \\
\frac{1-\mathrm{e}^{2 F_{2}}}{1+\mathrm{e}^{2 F_{2}}}\end{array}\right)$ & $O(1)$ \\
\hline
\end{tabular}

Table 4 Variable separation solution of the equivalent LLG equation without damping (see equation (60)). F satisfies (71); $F_{2}$ is the solution of (99)

\begin{tabular}{ll}
\hline Solutions I-II & Decay rate \\
\hline Solution I: $\mathrm{e}^{F} \exp \left[i\left(C_{1}+C_{2} \int r^{-n+1}\left(\mathrm{e}^{2 F}+1\right)^{2} \mathrm{e}^{-2 F} \mathrm{~d} r+C_{5} t\right)\right]$ & $\frac{1}{r^{n-1}},(n \geq 2) ;$ \\
Solution II: $\mathrm{e}^{F_{2}} \exp \left[i\left(C_{1}+C_{2} \int\left(\mathrm{e}^{2 F_{2}}+1\right)^{2} \mathrm{e}^{-2 F_{2}} \mathrm{~d} \bar{r}+C_{5} t\right)\right]$ & $O(1),(n=1)$ \\
\hline
\end{tabular}

\section{Conclusions}

We investigate a variable separation solution of the multidimensional ILLG equation and Schrödinger map equation. Based on the stereographic method, we deduce an equivalent ILLG equation, which can be solved exactly. Under the different ansatzs of the solutions, we obtain some explicit solutions of the modification system. The solution of this system can be changed into the solution of the ILLG equation. Hence, for the ILLG equation, we obtain two different solutions, which are all in a constant decay scale.

If $q=1$ and $\beta=0$, then the ILLG equation degenerates into the Schrödinger map equation. The situation of this equation is somewhat different from the ILLG equation: the solutions obtained are in implicit forms, which are determined by nonlinear ODEs. This brings some difficulties to our analysis of the decay behavior of the solutions. Using the some heuristic method, we obtain the asymptotic scale of the functions contained in the nonlinear system. So, the decay rate of the energy density of the solutions can be characterized. Clearly, we obtain two different solutions of the Schrödinger map equation, which adopt the same decay rate $O(1)$. However, the situation of its equivalent system is somewhat different: the decay rate of radial type solution is in an $r^{n-1}(n \geq 2)$ and $O(1)(n=1)$ scale, whereas the plane wave type solution is $O(1)$.

All solutions obtained in this paper are periodic in time or spatial direction. The solution of ILLG equation (or Schrödinger map equation) of type (4) just shows a periodic behavior in the time direction. Hence, if the periodic condition is imposed in the initial condition, then the ILLG equation and Schrödinger map equation contain some smooth solutions for some large initial values. In this study, the properties of the variable separation type solutions are clearer due to their straightforward and exact form. These solutions will be useful in explaining some nonlinear dynamics of stimulation in the inhomogeneous or homogeneous system that comes from the ferromagnet. 


\section{Funding}

This work is supported by TianYuan Special Fund of the National Natural Science Foundation of China (No. 11426068), Project for Young Creative Talents of Ordinary University of Guangdong Province (No. 2014KQNCX228), the PhD Start-up Fund of Natural Science Foundation of Guangdong Province (No. 2014A030310330), and Fund for Science and Technology of Guangzhou (No. 201607010352). The author is also grateful to the sponsorship from China Scholarship Council.

\section{Competing interests}

The authors declare that there are no competing interests.

\section{Authors' contributions}

All authors read and approved the final manuscript.

\section{Publisher's Note}

Springer Nature remains neutral with regard to jurisdictional claims in published maps and institutional affiliations.

Received: 24 July 2018 Accepted: 7 September 2018 Published online: 20 September 2018

\section{References}

1. Balakrishnan, R.: On the inhomogeneous Heisenberg chain. J. Phys. C, Solid State Phys. 15, 1305-1308 (1982)

2. Baleanu, D., Golmankhaneh, A.K., Golmankhaneh, A.K.: On electromagnetic field in fractional space. Nonlinear Anal., Real World Appl. 11, 288-292 (2010)

3. Bejenaru, I., lonescu, A., Kenig, C., Tataru, D.: Global Schrödinger maps in dimensions $d \geq 2$ : small data in the critical Sobolev spaces. Ann. Math. 173, 1443-1506 (2011)

4. Chang, N.-H., Shatah, J., Uhlenbeck, K.: Schrödinger maps. Commun. Pure Appl. Math. 53, 590-602 (2000)

5. Ding, Q.: Explicit blow-up solutions to the Schrödinger maps from $R^{2}$ to the hyperbolic 2-space $\mathcal{H}^{2}$. J. Math. Phys. 50, 103507 (2009)

6. Daniel, M., Porsezian, K. Lakshmanan, M.: On the integrability of the inhomogeneous spherically symmetric Heisenberg ferromagnet in arbitrary dimensions. J. Math. Phys. 35, 6498-6510 (1994)

7. Ding, S.J., Wang, C.Y.: Finite time singularity of the Landau-Lifshitz-Gilbert equation. Int. Math. Res. Not. (2007). https://doi.org/10.1093/imrn/rnm012

8. Gomez-Aguilar, J.F., Baleanu, D.: Schrödinger equation involving fractional operators with non-singular kernel. J. Electromagn. Waves Appl. 31, 752-761 (2018)

9. Guo, B.L., Yang, G.S.: Some exact nontrivial global solutions with values in unit sphere for two-dimensional Landau-Lifshitz equations. J. Math. Phys. 42, 5223-5227 (2001)

10. Huh, H.: Blow-up solutions of modified Schrödinger maps. Commun. Partial Differ. Equ. 33, 235-243 (2008)

11. He, X.M., Qian, A.X., Zou, W.M.: Existence and concentration of positive solutions for quasi-linear Schrödinger equations with critical growth. Nonlinearity 26, 3137-3168 (2013)

12. Kosevich, A., Ivanov, B., Kovalev, A.: Magnetic solitons. Phys. Rep. 194, 117-238 (1990)

13. Liu, X.G.: Concentration sets of the Landau-Lifshitz system and quasi-mean curvature flows. Calc. Var. Partial Differ. Equ. 27, 493-525 (2006)

14. Landau, L.D., Lifshitz, E.M.: On the theory of the dispersion of magnetic permeability in ferromagnetic bodies. Z. Sowjetunion 8 (1935). Reproduced in Collected Papers of L. D. Landau, Pergamon, New York, pp. 101-114 (1965)

15. Lin, J.Y., Lai, B.S., Wang, C.Y.: Global well-posedness of the Landau-Lifshitz-Gilbert equation for initial data in Morrey spaces. Calc. Var. Partial Differ. Equ. 54, 665-692 (2015)

16. Li, Y.X., Wang, Y.D.: Bubbling location for F-harmonic maps and inhomogeneous Landau-Lifshitz equations. Comment. Math. Helv. 81, 433-448 (2006)

17. Melcher, C:: Global solvability of the Cauchy problem for the Landau-Lifshitz-Gilbert equation in higher dimensions. Indiana Univ. Math. J. 61, 1175-1200 (2012)

18. Muslih, S.I, Agrawal, O.P., Baleanu, D.: A fractional Schrödinger equation and its solution. Int. J. Theor. Phys. 49, 1746-1752 (2010)

19. Morales-Delgado, V.F., Gómez-Aguilar, J.F., Baleanu, D.: A new approach to exact optical soliton solutions for the nonlinear Schrödinger equation. Eur. Phys. J. Plus 133, 1-18 (2018)

20. Morales-Delgado, V.F., Gómez-Aguilar, J.F., Taneco-Hernández, M.A., Baleanu, D.: Modeling the fractional non-linear Schrödinger equation via Liouville-Caputo fractional derivative. Optik 162, 1-7 (2018)

21. Merle, F., Raphaël, P., Radnianski, I.: Blowup dynamics for smooth data equivariant solutions to the critical Schrödinger map problem. Invent. Math. 193, 249-365 (2013)

22. Ma, X.N., Wang, P.H., Wei, W.: Constant mean curvature surfaces and mean curvature flow with non-zero Neumann boundary conditions on strictly convex domains. J. Funct. Anal. 274(1), 252-277 (2018)

23. Perelman, G.: Blow up dynamics for equivariant critical Schrödinger maps. Commun. Math. Phys. 330, 69-105 (2014)

24. Sun, Y., Liu, L.S., Wu, Y.H.: The existence and uniqueness of positive monotone solutions for a class of nonlinear Schrödinger equations on infinite domains. J. Comput. Appl. Math. 321, 478-486 (2017)

25. Sulem, P., Sulem, C., Bardos, C.: On the continuous limit for a system of classical spins. Commun. Math. Phys. 107, 431-454 (1986)

26. Van Den Berg, J.B., Williams, J.F.: (in-)stability of singular equivariant solutions to the Landau-Lifshitz-Gilbert equation. Eur. J. Appl. Math. 24, 921-948 (2013)

27. Yang, G.S., Chang, Q.S.: Limit behavior of solution for multi-dimensional Landau-Lifshitz equations with external magnetic field. Phys. Lett. A 318, 270-280 (2003)

28. Zhong, P.H., Wang, S., Chen, S.T.: Some periodic and blow-up solutions for Landau-Lifshitz equation. Mod. Phys. Lett. A 26, 2437-2452 (2011) 
29. Zhong, P.H., Wang, S., Zeng, M.: Two blowup solutions for the inhomogeneous isotropic Landau-Lifshitz equation. J. Math. Anal. Appl. 409, 74-83 (2014)

30. Zhong, P.H., Yang, G.S.: Finite time blowup of multidimensional inhomogeneous isotropic Landau-Lifshitz equation on a hyperbolic space. Comput. Math. Appl. 73, 433-449 (2017)

31. Zhang, X.G., Liu, L.S., Wu, Y.H., Cui, Y.J.: Entire blow-up solutions for a quasilinear $p$-Laplacian Schrödinger equation with a non-square diffusion term. Appl. Math. Lett. 74, 85-93 (2017)

32. Sun, F.L., Liu, L.S., Wu, Y.H.: Infinitely many sign-changing solutions for a class of biharmonic equation with $p$-Laplacian and Neumann boundary condition. Appl. Math. Lett. 73, 128-135 (2017)

33. Wang, P.H., Wang, X.J.: The geometric properties of harmonic function on 2-dimensional Riemannian manifolds. Nonlinear Anal. 103, 2-8 (2014)

34. Wang, P.H., Zhao, L.L.: Some geometrical properties of convex level sets of minimal graph on 2-dimensional Riemannian manifolds. Nonlinear Anal. 130, 1-17 (2016)

35. Han, M.A., Sheng, L.J., Zhang, X.: Bifurcation theory for finitely smooth planar autonomous differential systems. J. Differ. Equ. 264(5), 3596-3618 (2018)

36. Li, F.S., Gao, Q.Y.: Blow-up of solution for a nonlinear Petrovsky type equation with memory. Appl. Math. Comput. 274, 383-392 (2016)

37. Meng, F.W., Shao, J.: Some new Volterra-Fredholm type dynamic integral inequalities on time scales. Appl. Math. Comput. 223, 444-451 (2013)

38. Tian, H., Han, M.A.: Bifurcation of periodic orbits by perturbing high-dimensional piecewise smooth integrable systems. J. Differ. Equ. 263(11), 7448-7474 (2017)

39. Li, L.Z., Meng, F.W., Zheng, Z.W.: Oscillation results related to integral average technique for linear Hamiltonian systems. Dyn. Syst. Appl. 18, 725-736 (2009)

40. Li, L.Z., Meng, F.W., Zheng, Z.W.: Some new oscillation results for linear Hamiltonian systems. Appl. Math. Comput. 208, 219-224 (2009)

41. Zheng, Z.W.: Invariance of deficiency indices under perturbation for discrete Hamiltonian systems. J. Differ. Equ. Appl. 19(8), 1243-1250 (2013)

\section{Submit your manuscript to a SpringerOpen ${ }^{\circ}$ journal and benefit from:}

- Convenient online submission

- Rigorous peer review

- Open access: articles freely available online

- High visibility within the field

- Retaining the copyright to your article

Submit your next manuscript at $\gg$ springeropen.com 